\title{
Traditionelle Chinesische Medizin aus der Sicht von Schweizer Medizinstudierenden im Vorklinikum
}

\author{
Marko Nedeljković Brigitte Ausfeld-Hafter \\ Kollegiale Instanz für Komplementärmedizin KIKOM, Universität Bern, Schweiz
}

\section{Schlüsselwörter}

Komplementärmedizin, CAM - Traditionelle Chinesische Medizin, TCM · Umfrage · Medizinische Ausbildung · Medizinstudierende

\section{Zusammenfassung}

Hintergrund: Die Nachfrage nach komplementärmedizinischer Behandlung, insbesondere im Bereich der Traditionellen Chinesischen Medizin (TCM), hat im In- und Ausland im Verlauf der letzten beiden Jahrzehnte stetig zugenommen. Um zukünftige Ärzte kompetenter auf die Patientenbedürfnisse vorzubereiten, wurde von Fachpersonen aus der Forschung, klinischen Praxis, Bildung und Politik eine stärkere Integration der Komplementärmedizin (CAM) in bestehende Ausbildungsprogramme interdisziplinär diskutiert. Mit der vorliegenden Umfragestudie wurde bei Medizinstudierenden im Präklinikum sowohl deren Einstellung gegenüber, Erfahrung mit und Kenntnisstand zu der TCM in Erfahrung gebracht als auch abgeklärt, inwiefern das Interesse an einer Erweiterung ihres Pflicht-Curriculums um TCM-Unterrichtseinheiten vorhanden ist. Material und Methoden: 180 Medizinstudierende des zweiten Studienjahrs an der Universität Bern wurden gebeten, sich an der Fragebogenstudie zu beteiligen (Rücklaufquote 73,3\%). Ergebnisse: Für 3,8\% der Antwortenden ist die TCM der Schulmedizin ebenbürtig. Die grosse Mehrheit (76,3\%) sieht die TCM als eine zur westlichen Medizin komplementäre Behandlungsform. Für 9,2\% stellt die TCM eine primär auf dem Placeboeffekt beruhende Behandlungsform dar, und für 10,7\% gilt die TCM als veraltet. 46,6\% der Antwortenden befürworten die Aufnahme von zusätzlichen TCM-Veranstaltungen in das Pflichtstudium. $17,4 \%$ haben sich in der Vergangenheit mit TCM mit überwiegend positiver Rückmeldung behandeln lassen. Die Offenheit für TCM-Behandlungen bei jenen ohne Selbsterfahrung ist gross (76,1\%). Eigene TCM-Kenntnisse wurden von $76,5 \%$ als kaum vorhanden eingeschätzt. Schlussfolgerungen: Von den Medizinstudierenden im Vorklinikum wird der TCM trotz geringer Vorkenntnisse grosses Interesse entgegengebracht. Möglichkeiten zur stärkeren Einbindung der TCM in das bestehende Medizinstudium sind zu prüfen.

\section{Keywords}

Complementary and Alternative Medicine, CAM - Traditional Chinese Medicine, TCM · Survey · Medical Education · Medical Students

\section{Summary \\ Traditional Chinese Medicine from Swiss Preclinical Medical Students' Point of View}

Background: During the past two decades the demand for complementary and alternative medicine (CAM), and especially regarding Traditional Chinese Medicine (TCM), has continuously increased in Switzerland and abroad. Among experts representing the fields of research, clinical practice, education and politics it has been discussed whether the further integration of CAM into the existing curriculum would help to prepare future doctors meet the growing needs of their patients. A study was conducted to determine the attitudes, experiences and preconceptions of preclinical medical students regarding TCM as well as to assess their level of interest in making TCM a part of the compulsory curriculum. Materials and Methods: 180 second-year medical students of the University of Bern were asked to participate in the survey by filling in a questionnaire (return rate 73.3\%). Results: $3.8 \%$ of the respondents perceived the therapeutic benefit of TCM as equivalent to western medicine. The majority of participants, i.e. $76.3 \%$, saw TCM as a complementary treatment. 9.2\% attribute TCM to a placebo effect and $10.7 \%$ perceive TCM as antiquated. $46.6 \%$ of the respondents would welcome an integration of TCM in their compulsory curriculum. Most of the $17.4 \%$ of those students who had prior treatment experience with TCM expressed a positive opinion. The majority of those with no treatment experience (76.1\%) demonstrated a high degree of openness. $76.5 \%$ of the students judged themselves as having very limited prior knowledge of TCM. Conclusion: Despite sparse previous knowledge, second-year medical students show great interest in TCM. We suggest further investigation as to how TCM can be adequately integrated into the existing curriculum.

$\begin{array}{ll}\text { KARGER } & \text { @ 2012 S. Karger GmbH, Freiburg } \\ \text { 1015-0684/12/0242-0101\$38.00/0 } \\ \begin{array}{l}\text { Fax +49 761 452 07 14 } \\ \begin{array}{l}\text { Information@Karger.de } \\ \text { www.karger.com }\end{array}\end{array} & \begin{array}{l}\text { Accessible online at: } \\ \text { www.karger.com/szg }\end{array}\end{array}$

Marko Nedeljković

Kollegiale Instanz für Komplementärmedizin KIKOM

Universität Bern, Inselspital, Imhoof-Pavillon

3010 Bern, Schweiz

Tel. +41 31 632-9758, Fax -4262

marko.nedeljkovic@kikom.unibe.ch 


\section{Mots-clés}

Médecine complémentaire, MCA - Médecine traditionnelle chinoise, MTC · Sondage · Formation médicale · Etudiants en médecine

\section{Résumé}

La médecine traditionnelle chinoise vue par les étudiants suisses en médecine de premier cycle

Contexte: Ces vingt dernières années, l'intérêt des patients pour la médecine complémentaire, en particulier la médecine traditionnelle chinoise (MTC), n'a cessé de croître dans tous les pays. Afin de mieux préparer les futurs médecins à répondre avec compétence aux besoins des patients, des spécialistes de la recherche, de la pratique clinique, de la formation et de la sphère politique ont mené un débat interdisciplinaire sur l'intégration renforcée de la médecine complémentaire (MCA) dans les programmes de formation existants. Avec le présent sondage, on a mis en évidence l'attitude des étudiants en médecine de premier cycle vis-à-vis de la MTC, ainsi que leur expérience et leurs connaissances dans ce domaine. On a également étudié dans quelle mesure ces étudiants sont intéressés par un enrichissement de leur cursus obligatoire avec des unités d'enseignement consacrés à la TCM. Matériel et méthodes: 180 étudiants en deuxième année de médecine de I'Université de Berne ont été conviés à répondre au questionnaire de l'étude (73,3\% de taux de réponse). Résultats: 3,8\% des participants placent la MTC et la médecine généraliste conventionnelle sur un pied d'égalité. La grande majorité (76,3\%) envisage la MTC comme une forme de traitement venant complémenter la médecine occidentale. Selon 9,2\% des étudiants, la MTC repose principalement sur l'effet placebo et $10,7 \%$ d'entre eux considèrent qu'elle est obsolète. $46,6 \%$ des participants se montrent favorables à l'intégration de cours consacrés à la MTC à leur cursus de formation obligatoire. 17,4\% ont déjà eu recours à la MTC; avec des retours positifs dans la majorité des cas. Ceux qui n'ont pas expérimenté personnellement la TCM se montrent toutefois très ouverts à son égard (76,1\%). 76,5\% des étudiants ont estimé que leurs connaissances sur la TCM étaient quasiment inexistantes. Conclusions: Même s'ils ont très peu de connaissances sur la MTC, les étudiants en médecine de premier cycle ont manifesté un grand intérêt pour la MTC. II faut maintenant explorer les possibilités d'intégrer de façon plus proéminente la MTC dans le cursus médical actuel.

\section{Hintergrund}

Wie aus dem politischen Volksentscheid vom 17. Mai 2009 zur Abstimmung «Zukunft mit Komplementärmedizin» (Komplementärmedizin wird im Folgenden als CAM - Complementary and Alternative Medicine - bezeichnet) hervorgegangen ist, wird die CAM von der Mehrheit der Schweizer Bevölkerung als ein integraler Bestandteil des Schweizer Gesundheitswesens wahrgenommen [1, 2]. Die Nachfrage nach CAM-Behandlungen ist wie in anderen europäischen Ländern $[3,4]$ und in den Vereinigten Staaten [5] auch in der Schweiz relativ hoch. Ungefähr 30-50\% der Schweizer lassen sich jährlich mit CAM behandeln [6-8]. Auffallend dabei ist die relativ grosse Nachfrage seitens der Bevölkerung für Behandlungsformen der Traditionellen Chinesischen Medizin (Anmerkung: Diese wird nachfolgend mit TCM abgekürzt und ist als Überbegriff für alle Behandlungsmethoden, welche dieser Medizinrichtung zugehörig sind, zu verstehen. Wo möglich, werden differenzierte Angaben zu den einzelnen TCM-Behandlungsmethoden gemacht.). Der Schweizerischen Gesundheitsbefragung aus dem Jahre 2007 zufolge haben $6,6 \%$ der Schweizer Bevölkerung TCM-Behandlungen (4,9\% Akupunktur und 1,7\% chinesische Arzneitherapie) in Anspruch genommen [6]. Diese zählen neben der Homöopathie $(6,4 \%)$ und Osteopathie $(5,4 \%)$ zu den am häufigsten nachgefragten CAM-Methoden in der Schweiz [6]. Für den hohen Stellenwert der Akupunktur sprechen auch die Ergebnisse aus der Züricher Längsschnittstudie [7], wonach bei der untersuchten Stichprobe ( $\mathrm{n}=591$ ) aus dem Kanton Zürich die Nachfrage nach Akupunktur im Zeitraum von 1993 bis 1999 von 3,1 auf 10,7\% angestiegen ist. In der hausärztlichen Grundversorgung wird Akupunktur/TCM von 31,1\% der Hausärzte praktiziert und stellt nach Homöopathie (34\%) und Phytotherapie (34\%) die dritthäufigste CAM-Behandlungsform in Hausarztpraxen dar [9]. Was die Überweisungen zu CAMBehandlungen betrifft, werden PatientInnen von ihren Hausärzten am häufigsten zu TCM-Behandlungen (58,5\%), gefolgt von Behandlungen mit Homöopathie $(22,9 \%)$ und Anthroposophischer Medizin (10,3\%), überwiesen [9]. Auch in den Schweizer Spitälern stellen Akupunktur und TCM die beiden am häufigsten angebotenen CAM-Behandlungsformen dar [10]. Die inkonsistente Befundlage wissenschaftlicher Studien zur Wirksamkeit von TCMBehandlungsformen hat Anlass zu kontroversen Diskussionen gegeben [11-13]. Umso wichtiger ist eine evidenzgestützte Vermittlung der grundlegenden Kenntnisse über die in der Schweiz am meisten verbreiteten CAM-Behandlungsverfahren an die Medizinstudierenden. Durch eine solche sollen zukünftige Ärzte zumindest beratungskompetent auf die hohe Nachfrage in der Schweizer Bevölkerung nach CAM vorbereitet werden $[1,14]$. Wie an europäischen [15] und amerikanischen Universitäten [16] hielt CAM in den vergangenen Jahren auch an der Mehrzahl der Schweizer Universitäten Einzug in das Unterrichtsangebot der medizinischen Fakultäten. Auf Akupunktur/ TCM bezogen ergab eine im Jahre 2007 durchgeführte Befragung, dass diese an drei von fünf Universitäten in Form von fakultativen Unterrichtsveranstaltungen vertreten ist und von fortgeschrittenen Medizinstudierenden im sechsten Studienjahr als die wirksamste CAM-Behandlungsform betrachtet wird [17]. Weitere Studienergebnisse belegen, dass die Mehrheit der angehenden Ärzte neben Phytotherapie (65,8\%), Homöopathie (58,5\%) und klassischer Naturheilkunde (57\%) auch Akupunktur/TCM 
Einstellung und Interesse bezüglich TCM

1. Welche Aussage trifft auf Deine Einstellung zur Traditionellen Chinesischen Medizin (TCM) am besten zu?

«Ich betrachte die TCM als ...»

$\square$... eine der westlichen Medizin ebenbürtige Behandlungsform.

$\square$... eine Behandlungsform, welche die westliche Medizin komplementär sinnvoll zu ergänzen vermag.

$\square$... eine, gemessen an ihrer Entstehungszeit erstaunliche, heute aber nicht mehr zeitgemässe medizinische Behandlungsform.

$\square$... eine nichtmedizinische Behandlungsform, deren Wirkung primär dem Placeboeffekt zuzuschreiben ist.

2. Bist Du im Rahmen Deines Medizinstudiums in Kontakt mit der TCM gekommen? (hier sind mehrere Antworten möglich)

$\square \mathrm{Ja}$, im Rahmen einzelner Unterrichtslektionen in Pflichtveranstaltungen

$\square \mathrm{Ja}$, im Rahmen von einem Wahlpraktikum.

$\square \mathrm{Ja}$, im Rahmen von fakultativen Vorlesungen.

$\square \mathrm{Ja}$, im Rahmen von TCM-Weiterbildungskursen für Ärzte und Studierende.

$\square$ Nein.

3. Bist Du an (zusätzlichen) Veranstaltungen zur TCM im Rahmen des Pflichtstudiums interessiert?

$\square \mathrm{Ja} / \square$ Nein

4. Könntest Du Dir vorstellen, später in der Praxis Behandlungsformen und -prinzipien der TCM anzuwenden?

$\square \mathrm{Ja} / \mathrm{Nein} / \mathrm{Das}$ weiss ich noch nicht

5. Könntest Du Dir eine ärztliche TCM-Weiterbildung in Akupunktur vorstellen?

$\square \mathrm{Ja}$, ich könnte mir eine ärztliche Weiterbildung zum Fähigkeitsausweis in Akupunktur vorstellen. $\square$ Nein, voraussichtlich werde ich nach dem Studium keine Akupunkturweiterbildung absolvieren. $\square$ Das weiss ich noch nicht.

\section{Erfahrung mit TCM}

6. Warst Du selbst schon einmal in einer TCM-Behandlung?

$\square$ Nein. Ich kann mir aber durchaus vorstellen, mich mit TCM behandeln zu lassen.

$\square$ Nein. Ich kann mir auch nicht vorstellen, mich jemals mit TCM behandeln zu lassen.

$\square \mathrm{Ja}$, einmal.

$\square \mathrm{Ja}$, mehrere Male.

7. Falls Du die Frage 6 mit «Ja» beantwortet hast, wie war/en Deine Erfahrung/en mit der/n TCM-Behandlung/en?

$\square$ Durchwegs positiv, würde mich jederzeit wieder mit TCM behandeln lassen.

$\square$ Hatte gemischte Erfahrungen gemacht.

$\square$ Hatte keine spürbaren Symptomverbesserungen erlebt.

$\square$ Schlecht, mache ich nie wieder

\section{TCM-Kenntnisse}

8. Welche Aussage trifft auf Deinen Kenntnisstand über die TCM am besten zu?

$\square$ Über die TCM weiss ich so gut wie gar nichts.

$\square$ Ich verfüge über Grundkenntnisse der TCM.

$\square$ Ich weiss über die wesentlichen Theorien und Begrifflichkeiten der TCM Bescheid.

Abb. 1. Fragebogen.

$\square$ Ich verfüge aufgrund von über ein solides Fachwissen im Bereich der TCM 
(75,3\%) als Unterrichtsinhalt im Medizinstudium als wünschenswert angibt, jedoch nur knapp jeder Dritte von ihnen den Rahmen einer Pflichtveranstaltung für CAMInhalte als passend erachtet und lediglich 23,3\% der Antwortenden den CAM-Unterricht im Vorklinikum befürworteten [18]. Angenommen wird, dass das Vermögen der Studierenden, mehr über CAM in Erfahrung zu bringen, neben Form und Inhalt des universitären CAM-Lehrangebots auch von weiteren studentenseitigen Faktoren wie beispielsweise der allgemeinen Einstellung zur CAM, dem Vorhandensein von Vorkenntnissen über und Selbsterfahrungen mit CAM und der Eigenmotivation zum Lernen und Anwenden von CAM mit beeinflusst wird [19]. Aus vergleichenden Studien ist unterdessen bekannt, dass unter den Medizinstudierenden die Einstellung zur CAM und die Motivation, mehr über CAM in Erfahrung zu bringen, mit fortschreitendem Studium abnehmen kann [20-23]. Mit der vorliegenden Umfragestudie wurde daher beabsichtigt, die bestehende Datenlage in einem ersten Schritt um die TCM-bezogenen Angaben von Medizinstudierenden im Präklinikum zu erweitern. Nebst deren Einstellung zur und deren Lerninteresse an der TCM wurden auch deren eigene Erfahrungen mit, deren Wissen über die TCM sowie deren längerfristige Motivation, sich später durch ärztliche Fortbildungen Behandlungskompetenzen in der TCM anzueignen, erfragt. Letzteres wurde insbesondere in Anbetracht der Tatsache, dass nur die Hälfte der Schweizer Hausärzte, die CAM-Behandlungen anbieten, auch über einen entsprechenden FMH-anerkannten Fähigkeitsausweis (FMH - Foederatio Medicorum Helveticorum - Verbindung Schweizer Ärztinnen und Ärzte) verfügt [9], in den Fragekatalog aufgenommen.

\section{Material und Methoden}

Stichprobe und Datenerhebung

Die Befragung wurde mit dem Einverständnis des Institutes für medizinische Lehre der Universität Bern bei Medizinstudierenden im zweiten Studienjahr $(\mathrm{n}=180)$ an der Universität Bern durchgeführt. Während einer Vorlesungspause bei einer Pflichtveranstaltung wurde den Studierenden ein Fragebogen zur schriftlichen Beantwortung vorgelegt.

\section{Datenanalyse}

Die von den 132 Antwortenden erhaltenen Fragebogendaten wurden mit dem Statistikprogramm PASW Statistics 18.0 (IBM SPSS Statistics, Somers, NY, USA) deskriptiv ausgewertet. Die Daten aus den partiell ausgefüllten Fragebögen $(n=3)$ wurden in der statistischen Datenauswertung mitberücksichtigt, da sie jeweils nur einen fehlenden Wert aufwiesen.

\section{Fragebogen}

Der Fragebogen bestand aus sieben Einfachauswahl-Fragen und einer Mehrfachauswahl-Frage. Vor Einsatz des Fragebogens wurde dieser im Rahmen eines Pre-Tests von einer Gruppe bestehend aus 10 Studierenden unterschiedlicher Fachrichtungen auf dessen Verständlichkeit hin überprüft. Die gestellten Fragen und Antwortmöglichkeiten sind in Abbildung 1 aufgeführt.

\section{Ergebnisse}

Eigenschaften der befragten Studentenpopulation Zum Befragungszeitpunkt am 27. Januar 2010 waren 212 Medizinstudierende, 130 Studentinnen (61,3\%) und 82 Studenten (38,7\%), im zweiten Studienjahr immatrikuliert. Von diesen waren 180 an der Pflichtveranstaltung zum Erhebungszeitpunkt anwesend.

132 Fragebögen wurden zurückgegeben. Damit betrug die Rücklaufquote $73,3 \%$. Von den abgegebenen Fragebögen waren 129 vollständig und 3 mit jeweils einer fehlenden Angabe ausgefüllt. Insgesamt haben sich 62,3\% der im zweiten Jahr immatrikulierten Medizinstudierenden an der Umfrage beteiligt, d.h. $82(62,1 \%)$ Studentinnen und 50 (37,9\%) Studenten. Das Geschlechterverhältnis bei den Antwortenden stimmt mit jenem der Untersuchungspopulation überein. Das Durchschnittsalter der Antwortenden betrug 21,6 Jahre (Standardabweichung = 2,3).

\section{Einstellung}

Die Auswertung der Antworten ( $\mathrm{n}=131$ ) hat ergeben, dass die Mehrheit (76,3\%) die TCM als eine die westliche Medizin sinnvoll ergänzende therapeutische Richtung betrachtet. Weitere 3,8\% der Studierenden nehmen die TCM als der westlichen Medizin ebenbürtig wahr. 9,2\% der Antwortenden betrachten die TCM als eine Behandlungsform, die primär über den Placeboeffekt zu erklären ist. Für 10,7\% gilt sie als eine, gemessen an ihrer Entstehungszeit erstaunliche, heute aber nicht mehr zeitgemässe medizinische Behandlungsform (Abb. 2).

\section{Interesse}

Nur 6,8\% $(n=9)$ der Antwortenden sind in ihrem bisherigen Medizinstudium im Rahmen von fakultativen Veranstaltungen in Kontakt mit der TCM gekommen. 46,6\% der Antwortenden befürworten die Aufnahme von zusätzlichen TCM-Veranstaltungen in das Pflichtstudium.

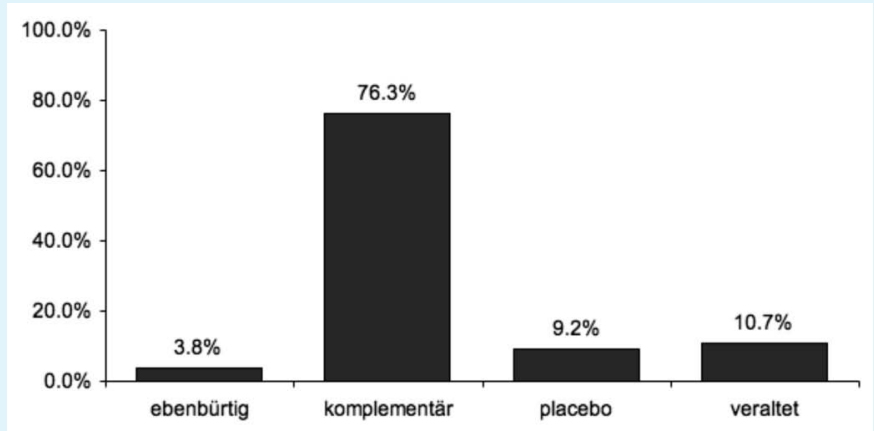

Abb. 2. Einstellung zur TCM $(\mathrm{n}=131)$. 
Wie aus Abbildung 3a ersichtlich wird, ist die grosse Mehrheit der Befürwortenden (96,7\%) gegenüber der TCM positiv eingestellt. Auch von den Ablehnenden wurde mit einer knappen Zweidrittelmehrheit $(65,2 \%)$ eine der TCM zugewandte Haltung bekundet.

25,8\% der Antwortenden können sich vorstellen, TCM später in der Praxis anzuwenden, und nehmen die TCM mit $11,8 \%$ als ebenbürtig, mit $82,4 \%$ als komplementär zur westlichen Medizin und mit 5,9\% als veraltet wahr. 41,7\% können dies zurzeit noch nicht beurteilen, sind der TCM gegenüber jedoch ebenfalls mehrheitlich aufgeschlossen. Jene Antwortende, die sich eine Integration der TCM in ihrer zukünftigen ärztlichen Tätigkeit nicht vorstellen können (32,5\%), haben gegenüber der TCM gemischte Einstellungen (Abb. 3b).

34,8\% der Antwortenden geben an, dass für sie der Besuch einer ärztlichen Weiterbildung in Akupunktur vorstellbar sei, und sind der TCM dementsprechend mehrheitlich aufgeschlossen (93,5\%). 18,9\% sind sich darüber noch nicht schlüssig, bekunden aber ebenfalls eine der TCM deutlich zugewandte Haltung (96,0\%). Die Einstellungen zur TCM seitens der Antwortenden, die keine
Weiterbildung in Akupunktur zu besuchen beabsichtigen, sind gemischt, mehrheitlich jedoch der TCM gegenüber aufgeschlossen (Abb. 3c).

\section{Selbsterfahrung}

82,6\% der Antwortenden gaben an, sich noch nie einer TCM-Behandlung unterzogen zu haben. Von ihnen können sich aber 76,1\% durchaus vorstellen, sich in Zukunft mit TCM behandeln zu lassen. Von den $17,4 \%$ der Studierenden, die sich schon ein- oder mehrmals mit TCM haben behandeln lassen, wurde die Behandlung von 43,5\% positiv erlebt. 30,4\% konnten keinen spürbar positiven Effekt bemerken. Die restlichen $26,1 \%$ berichten von gemischten, jedoch keinen ausschliesslich schlechten Erfahrungen. Wie in Abbildung 4 veranschaulicht, sind Medizinstudierende mit TCM-Selbsterfahrung dieser Behandlungsform gegenüber kritischer eingestellt als jene ohne TCM-Selbsterfahrung.

\section{Kenntnisstand}

76,5\% der Antwortenden haben ihrer Selbsteinschätzung zufolge so gut wie gar keine Kenntnisse über die TCM. 19,7 bzw. 3,8\% gaben an, jeweils über TCM-Grundkenntnisse
Abb. 3. Interesse an der TCM. a Aufnahme zusätzlicher TCM-Veranstaltungen ins Pflichtstudium ( $\mathrm{n}=131)$ ? b Zukünftige Anwendung von TCM in der Praxis $(\mathrm{n}=132)$ ? c Besuch ärztlicher Weiterbildung in Akupunktur $(\mathrm{n}=132)$ ? a

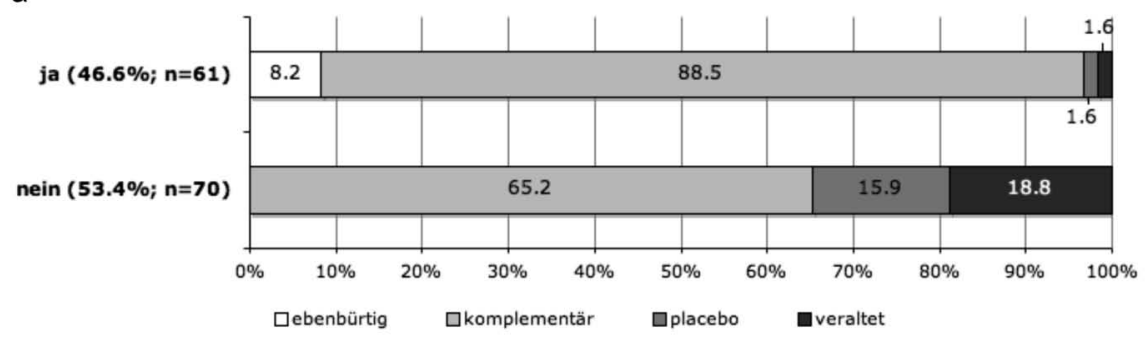

b

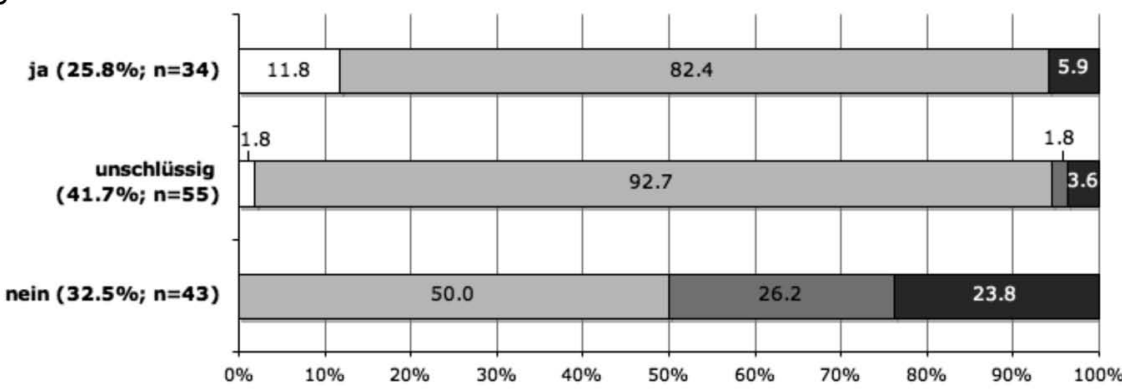

c

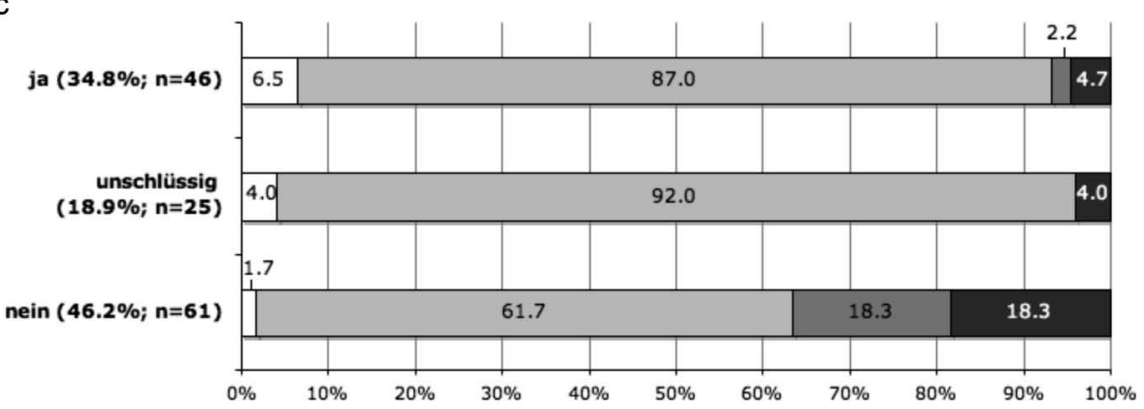




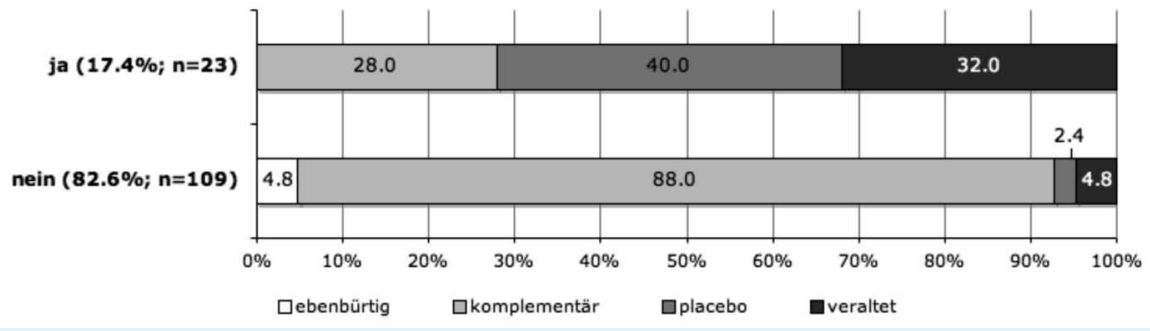

Abb. 4. Selbsterfahrung mit TCM vorhanden $(\mathrm{n}=132)$ ? bzw. über gute TCM-Kenntnisse zu verfügen. Sich ein umfassendes TCM-Fachwissen angeeignet zu haben, wurde von keiner Person vermerkt.

\section{Diskussion}

Die vorliegenden Befragungsergebnisse belegen eindeutig das Vorhandensein einer ausgeprägten Offenheit gegenüber der TCM seitens der Medizinstudierenden im Vorklinikum $(80,1 \%)$. Knapp die Hälfte der Studierenden $(46,6 \%)$ ist an einer zusätzlichen Erweiterung des Pflichtstudiums um Lerninhalte der TCM interessiert. Im Vergleich dazu wurde dieses Interesse von weniger als einem Drittel $(31,4 \%)$ der Medizinstudierenden im sechsten Studienjahr bekundet [18]. Denkbar wäre, dass das Interesse an der TCM und an CAM im Allgemeinen bei den Studierenden im Verlauf des allopathisch ausgerichteten Medizinstudiums abnimmt [20, 21]. Die Ergebnisse der amerikanischen Längsschnittstudie von Lie und Boker [19] legen jedoch nahe, dass die Grundeinstellung der Studierenden zur CAM relativ robust ist und es sich bei einem Unterschied zwischen den Studienjahren um einen Kohorteneffekt handelt. Ein solcher würde in diesem Falle ein nachhaltiges Interesse an der TCM bei der befragten Stichprobe implizieren. Dies wurde insofern bestätigt, da jeder vierte Antwortende angibt, sich vorstellen zu können, die TCM im späteren Praxisalltag zu integrieren, und jeder dritte Antwortende den Besuch einer ärztlichen Weiterbildung in Akupunktur in Erwägung zieht. Denkbar ist, dass die Studierenden an der Akupunktur als separate Behandlungsmethode mehr interessiert sind als an der TCM im Sinne eines umfassenden Medizinsystems.

Da die Akupunktur seit 1984 als einzige ärztliche CAM-Methode von den Grundversicherungen abgedeckt wird, wäre ein allenfalls stärkeres Interesse an dieser Behandlungsmethode möglicherweise durch deren grössere Akzeptanz seitens beispielsweise der Behörden oder Krankenkassen zu erklären. Welchen Einfluss die Wiederaufnahme der chinesischen Arzneitherapie sowie der weiteren vier ärztlichen CAM-Methoden Homöopathie, Anthroposophische Medizin, Neuraltherapie und Phytotherapie ab dem 1. Januar 2012 in die Grundversicherung auf die CAM-bezogenen Lerninteressen der Medizinstudierenden haben wird, ist Gegenstand zukünftiger Forschungsarbeiten. Dass sich mehr Medizinstudierende für TCM-bezogene Pflichtveranstaltungen geäussert haben $(46,6 \%)$ als für den Besuch einer ärztlichen Akupunkturweiterbildung $(34,8 \%)$ oder für eine zukünftige Integration der TCM in die eigene Praxistätigkeit (25,8\%), ist gut nachvollziehbar, da nicht jeder gewünschte oder für als notwendig erachtete Unterrichtsinhalt gleich zur Fachspezialisierung führen muss. Auch von den fortgeschrittenen Studierenden wird zu 92,5\% von CAM-bezogenen Lehrveranstaltungen eine Förderung der eigenen Meinungsbildung über CAM gewünscht. Im Vergleich dazu wird eine professionelle CAM-Ausbildung im Rahmen des Medizinstudiums von den wenigsten $(15,4 \%)$ erwartet [18]. Insofern dürften entsprechend ausgerichtete Lehrveranstaltungen für die Studierenden von breitem Interesse sein und für angehende Spezialisten eine sinnvolle Vorbereitung auf die praxisorientierten ärztlichen Weiterbildungsmodule zur Erlangung des Fähigkeitsausweises darstellen. Die Einstellungen gegenüber der TCM seitens der verschiedenen Untergruppen berücksichtigend, weisen die vorliegenden Angaben auf ein deutliches Vorhandensein von Offenheit für und starkem Interesse an einer Auseinandersetzung mit der TCM hin (Abb. 3). Inwiefern sich daraus stichhaltige Rückschlüsse hinsichtlich der Anzahl der zukünftig in TCM auszubildenden Ärzte ziehen lassen, ist jedoch angesichts der verbleibenden mehrjährigen Studiendauer der Befragten ungewiss. Ausserdem ist nicht anzunehmen, dass die Studierenden bereits zum jetzigen Zeitpunkt über die Anforderungen zur Erlangung eines Fähigkeitsausweises in TCM orientiert sind, was den Entscheid über die Aufnahme einer TCM-Weiterbildung nebst weiteren Faktoren mit beeinflussen kann. Gleichwohl zeugen diese Resultate von einem praxisbezogenen Interesse, was hinsichtlich der Sicherstellung des in TCM auszubildenden Ärztenachwuchses als ein positives Zeichen aufgefasst werden darf.

Da einzelne Unterrichtseinheiten zur TCM an der Universität Bern seit Jahren in Form von Wahlpraktika für die Studierenden im Vorklinikum angeboten werden, wurden diese nur zu ihrem Interesse an zusätzlichen TCM-Pflichtveranstaltungen befragt. Angesichts des bereits dicht gedrängten Stundenplans im Medizinstudium ist es nicht ausgeschlossen, dass sich einzelne Studierende trotz grund- 
sätzlich vorhandenem Interesse an der TCM gegen die Einführung von zusätzlichen TCM-bezogenen Lehrveranstaltungen geäussert haben, um einer Überlastung des Lernstoffs entgegenzuwirken. Ergebnisse der vorliegenden Umfragestudie unterstützen diese Annahme, zumal beachtliche 65,2\% der Ablehnenden die TCM gleichwohl als eine zur westlichen Medizin komplementäre Behandlungsform wahrnehmen (Abb. 3a). Im Rahmen von weiterführenden Untersuchungen wäre dieses Item zur Unterrichtsnachfrage mit differenzierteren Antwortmöglichkeiten zu ergänzen. Neben dem Interesse an zusätzlichen TCMbezogenen Pflichtveranstaltungen sollte auch der Bedarf an der Integration von TCM-Lerninhalten in den bestehenden Unterrichtsveranstaltungen wie auch der Bedarf nach mehr TCM-Wahlpraktika abgeklärt werden.

Die Angaben zur Selbsteinschätzung weisen darauf hin, dass gut Dreiviertel der Befragten kaum über TCMFachkenntnisse verfügen. Eine solche Diskrepanz zwischen einem hohen Interesse an und einem niedrigen selbstberichteten Kenntnis- bzw. Erfahrungsstand mit CAM und TCM konnte in vorgängigen Studien ebenfalls beobachtet werden [24-26]. Als plausible Erklärungen hierfür sind der fehlende Kontakt mit der TCM während des bisherigen Medizinstudiums (bei 93,2\% der Antwortenden) und das Fehlen von Selbsterfahrung (bei 82,6\% der Antwortenden) $\mathrm{zu}$ nennen. Interessanterweise bekunden Medizinstudierende mit TCM-Selbsterfahrung eine gegenüber der TCM kritischere Haltung im Vergleich zu jenen ohne TCM-Selbsterfahrung (Abb. 4). Möglicherweise wurde von der ersten Untergruppe initial der Wirksamkeit von TCM eine zu hohe Erwartungshaltung entgegengebracht, die nach erfolgter Selbsterfahrung deren Einstellung zur TCM beeinflusst haben mag. Längsschnittstudien wären zur Klärung dieses Sachverhaltes erforderlich.

In Anbetracht der Beteiligung von 62,1\% $(\mathrm{n}=132)$ der Medizinstudierenden des zweiten Studienjahres an der Universität Bern darf die Aussagekraft der vorliegenden Studentenbefragung bezüglich der Untersuchungspopulation als hoch betrachtet werden. Anzunehmen ist, dass der im Mai 2009 gefällte Volksentscheid zur Verankerung der CAM in der Bundesverfassung bei den Medizinstudierenden zu einer erhöhten Aufmerksamkeit bezüglich der Relevanz von CAM in der Medizin beigetragen hat. Inwiefern die vorliegenden Studienergebnisse auch auf die übrigen Schweizer Medizinstudierenden im Vorklinikum übertragen werden können, bleibt fraglich, zumal die TCM an den Schweizer Universitäten unterschiedlich stark vertreten ist [17] und dies gegebenenfalls einen Einfluss auf die Wahl des Studienortes haben kann. Da dieser Faktor zur Stichprobenselektivität bei der vorliegenden Arbeit nicht berücksichtigt wurde, sind die Studienergebnisse in erster Linie für die Universität Bern repräsentativ. Eine weitere Einschränkung der präsentierten Studienergebnisse ist deren thematische Eingrenzung auf die TCM, wodurch die Streubreite der Antworten niedriger ist, als dies bei einem erweiterten Antwortspektrum der Fall wäre. Direkte Vergleiche zu anderen CAM-Methoden können aufgrund der vorliegenden Datenlage keine gezogen werden; dafür geben die Ergebnisse klare Auskunft, dass Berner Medizinstudierende im zweiten Studienjahr der TCM gegenüber eine grosse Offenheit entgegenbringen und ein beachtliches Interesse an TCM-bezogenen Unterrichtsveranstaltungen zeigen. Inwiefern die bereits eingeführten TCM-Wahlpraktika für Studierende im Vorklinikum deren Nachfrage an TCM-Unterricht zu decken vermag oder die Einführung zusätzlicher Unterrichtsformen in Erwägung zu ziehen ist, soll durch weiterführende Untersuchungen abgeklärt werden.

\section{Dank}

Die Autoren danken herzlich den Medizinstudierenden im ersten Studienjahr Tina Rohrbach, Ariane Meister, Vera Guyer, Anita Maurer und Renato Comazzi für ihre engagierte Unterstützung bei der Datenerhebung im Rahmen ihres TCM-Wahlpraktikums und allen Medizinstudierenden im zweiten Studienjahr, die sich an der Umfrage beteiligt haben.

\section{Disclosure Statement}

Keine Interessenkonflikte.

\section{Literatur}

1 Albonico $\mathrm{H}$ : Komplementärmedizin in der Bundesverfassung der Schweiz. Schweiz Zschr Ganzheitsmedizin 2009;21:294-299.

-2 Saller R: Komplementärmedizin in der Verfassung: Die Schweizer Bevölkerung hat entschieden. Forsch Komplementmed 2009;16:216.

-3 Hanssen B, Grimsgaard S, Launsø L, Fønnebø V, Falkenberg T, Rasmussen NK: Use of complementary and alternative medicine in the Scandinavian countries. Scand J Prim Health Care 2005;23:57-62.
4 Fox P, Coughlan B, Butler M, Kelleher C: Complementary alternative medicine (CAM) use in Ireland: a secondary analysis of SLAN data. Complement Ther Med 2010;18:95-103.

5 Tindle HA, Davis RB, Phillips RS, Eisenberg DM: Trends in use of complementary and alternative medicine by US adults: 1997-2002. Alt Ther Health Med 2005;11:42-49.

6 Bundesamt für Statistik: Schweizerische Gesundheitsbefragung 2007 - Standardtabellen. Neuchâtel, BFS, 2009.
Rössler W, Lauber C, Angst J, Haker H, Gamma A, Eich D, Kessler RC, Ajdacic-Gross V: The use of complementary and alternative medicine in the general population: results from a longitudinal community study. Psychol Med 2007;37:73-84.

$>$ Wolf U, Maxion-Bergemann S, Bornhoft G, Matthiessen PF, Wolf M: Use of complementary medicine in Switzerland. Forsch Komplementmed 2006;13(suppl 2):4-6. 
9 Déglon-Fischer A, Barth J, Ausfeld-Hafter B: Komplementärmedizin in Schweizer Praxen der Grundversorgung. Forsch Komplementmed 2009;16:251-255.

10 Widmer M, Donges A, Wapf V, Busato A, Herren S: The supply of complementary and alternative medicine in Swiss hospitals. Forsch Komplementmed 2006;13:356-361.

11 Manheimer E, Wieland S, Kimbrough E, Cheng K, Berman BM: Evidence from the Cochrane Collaboration for Traditional Chinese Medicine therapies. J Altern Complement Med 2009;15:1001-1014

12 Singh S, Ernst, E: Alternative Medicine on Trial: Trick or Treatment? London, Bantam Press, 2008.

13 Maxion-Bergemann S, Bornhoft G, Sonderegger E, Renfer A, Matthiessen PF, Wolf U: Traditional Chinese Medicine (phytotherapy): Health Technology Assessment report - selected aspects. Forsch Komplementmed 2006;13(suppl 2):30-41.

14 Rist L, Schwabl H: Komplementärmedizin im politischen Prozess. Forsch Komplementmed 2009;16:76-78.

15 Varga O, Márton S, Molnár P: Status of complementary and alternative medicine in European medical schools. Forsch Komplementmed 2006;13:41-45.

16 Pearson NJ, Chesney MA: The CAM Education Program of the National Center for Complementary and Alternative Medicine: an Overview. Acad Med 2007;82:921-926.

17 Nicolao M, Täuber MG, Marian F, Heusser P: Complementary medicine courses in Swiss medical schools: actual status and students' experience. Swiss Med Wkly 2010;140:44-51.

18 Nicolao M, Täuber MG, Heusser P: How should complementary and alternative medicine be taught to medical students in Switzerland? A survey of medical experts and students. Med Teach 2010;32:50-55.

19 Lie D, Boker, J: Development and validation of the CAM Health Belief Questionnaire (CHBQ) and CAM use and attitudes amongst medical students. BMC Med Educ 2004;4:2.

20 Riccard CP, Skelton M: Comparative analysis of 1st, 2nd, and 4th year MD students' attitudes toward Complementary Alternative Medicine (CAM). BMC Res Notes 2008;1:84.

21 DeSylvia D, Stuber M, Fung CC, Bazargan-Hejazi S, Cooper E: The knowledge, attitudes and usage of complementary and alternative medicine of medical students. Evid Based Complement Alternat Med 2008; DOI: 10.1093/ecam/nen075.

-22 Furnham A, McGill C: Medical students' attitudes about complementary and alternative medicine. J Altern Complement Med 2003;9:275-284.

23 Greenfield SM, Innes MA, Allan TF, Wearn AM: First year medical students' perceptions and use of complementary and alternative medicine. Complement Ther Med 2002;10:27-32.

24 Kolkman E: CAM-education in the medical curriculum: vision of medical students. Eur J Integr Med 2009;1:195.

-25 Chaterji R, Tractenberg RE, Amri H, Lumpkin M, Amorosi SB, Haramati A: A large-sample survey of first- and second-year medical student attitudes toward complementary and alternative medicine in the curriculum and in practice. Altern Ther Health Med 2007;13:30-35.

26 Witt CM, Brinkhaus B, Willich SN: Teaching complementary and alternative medicine in a reform curriculum. Forsch Komplementmed 2006; 13:342-348. 